Topiques, études satoriennes

Topoï Studies, Journal of the SATOR

\title{
Le Mentorat féminin dans les contes de fées : de ruses en ruses
}

\section{Marianne Legault}

Volume 4, 2018

Maître-disciple : une relation topique

URI : https://id.erudit.org/iderudit/1074720ar

DOI : https://doi.org/10.7202/1074720ar

Aller au sommaire du numéro

Éditeur(s)

SATOR, Société d'Analyse de la Topique Romanesque d'Ancien Régime

ISSN

2369-4831 (numérique)

Découvrir la revue

Citer cet article

Legault, M. (2018). Le Mentorat féminin dans les contes de fées : de ruses en ruses. Topiques, études satoriennes / Topoï Studies, Journal of the SATOR, 4, 1-19. https://doi.org/10.7202/1074720ar
Résumé de l'article

Cet article examine les différentes formes de mentorats féminins dans trois contes de fées du Grand Siècle: Riquet à la Houppe, L’Adroite princesse ou les aventures de Finette, et L'Enchanteur. On y trace le rôle du mentorat dans la relation conteuses-lectrices, ainsi que son influence sur le refus des contraintes domestiques et sociales imposées sur les femmes. Le mentorat féminin, qui apparaît chez ces conteuses dans des maximes, dans un appel à l'écriture, ou dans la conclusion de l'intrigue, sert à dénoncer les hommes et à encourager les femmes tant à la production du genre merveilleux qu’à la liberté de conduite féminine. 
Marianne Legault

\section{Le Mentorat féminin dans les contes de fées : De ruses en ruses}

Le conte de fées a toujours incorporé une part de mentorat. Après tout, dès sa formation dans l'espace du folklore pré-médiéval, le conte de fées existait principalement pour divertir mais aussi pour instruire son audience. Grâce à cet outil de tradition orale, les nourrices et gouvernantes tissaient des récits au cœur desquels s'énonçaient de nombreux conflits d'enfance suivis de leurs résolutions, normalement assurées à la fin du conte. Ces histoires pouvaient ainsi préparer les enfants et les adolescents en les aidant à faire face à la possibilité d'éventuels conflits, illustrés sous formes de luttes internes et généralement d'ordre moral. Dans le déploiement de cette quête vers une identité adulte, le conte de fées, entremêlant le merveilleux à la mythologie ${ }^{1}$, devenait alors une sorte de carte routière menant le héros ou l'héroïne, après quelques étapes difficiles, à la récompense ultime, c'est à dire sa maturité. Essentiellement maitrisée par les femmes, la performance orale du conte souligne bien le lien étroit que celui-ci entretenait avec le domaine du féminin dès sa présence avant l'ère médiévale ${ }^{2}$. On ne saurait dès lors ignorer le tableau du double mentorat qui s'offre à notre imagination: la gouvernante raconte son récit merveilleux à un jeune public attentif, tandis que son conte illustre en lui-même une leçon morale.

Plusieurs siècles s'écoulent. Le genre du merveilleux renait dans les dernières années du dixseptième siècle avec un succès éblouissant en France. Mais le conte de fées a désormais étendu son empire jusqu'ici restreint à l'oralité pour devenir un genre littéraire et produit surtout par des

\footnotetext{
${ }^{1}$ Jack Zipes, Fairy Tales and the Art of Subversion: The Classical Genre for Children and the Process of Civilization, 1983, p. 7.

${ }^{2}$ Dans leurs études respectives, Mary Elizabeth Storer et Jack Zipes ont tous deux noté la tradition folklorique orale et essentiellement féminine du conte (M. E. Storer, Un épisode littéraire de la fin du XVII siècle. La mode des contes de fées (1685-1700), 1928, p. 15). Pour Zipes toutefois, le lien entre le conte de fées et la femme dépassait alors les limites de la simple narration. Zipes remarque en effet que le contenu du genre privilégiait également le rôle de la femme en mettant en scène des récits encrés dans la mythologie féminine ou encore en illustrant des aventures où les personnages féminins dominaient l'action. L'arrivée à la fin du Moyen Âge d'un goût marqué pour la chasse aux sorcières auraient, selon Zipes, initié une "patriarcalisation " du genre et éradiqué la dominance féminine du conte de fées, du moins jusqu'aux dernières années du dix-septième siècle (Fairy Tales and the Art of Subversion, op. cit., p. 17-18; également cité dans Marianne Legault, Narrations déviantes: L'intimité entre femmes dans l'imaginaire français du dix-septième siècle, 2008, p. 184).
} 
femmes, qui, entre 1690 et 1715, sont responsables du deux tiers de sa production ${ }^{3}$. D'une certaine façon, la progression du conte oral au conte littéraire qui s'effectue alors en France souligne de nouveau le lien étroit entre conte de fées et mentorat féminin. Car dans ce déferlement du merveilleux que subit la fin de siècle, les conteuses se constituent en assemblées ou en salons, et se consultent à propos de leurs créations ${ }^{4}$. De narrations (ou «mitonnages ») à rédactions, ces rassemblements de conteuses, nous rappelle Charlotte Trinquet, donnent naissance à nombres contes de fées littéraires, dont ceux de Madame d'Aulnoy ${ }^{5}$. Les concepts de complicité féminine et de production littéraire sont ainsi étroitement liés au thème du mentorat dans le conte de fées féminin.

Suite à son expansion d'un genre essentiellement oral à une vogue littéraire, le conte subit aussi un deuxième changement d'importance, soit la transformation d'une audience jadis centrée sur l'enfant à celle dorénavant focalisée sur un public adulte, élite, généralement féminin, et mondain. Comme l'a déjà noté Patricia Clancy, «Perrault and the women writers who established the fairy tales among the aristocracy in France in the 1690s [...] all focused on the manners, mores, speech and sensibility that was becoming French civilité $\gg{ }^{6}$. Aussi, avec son emphase sur le bon goût, la bienséance et les bonnes manières, l'aspect guide moral du conte de

\footnotetext{
${ }^{3}$ Gabrielle Verdier, «Approaches to the Literary Fairy Tale », dans Marie-France Hilgar (éd.), Actes de Las Vegas : théorie dramatique, Théophile de Viau, les contes de fées, 1991, p. 141-146 (p. 144-145).

${ }^{4}$ Nombreux sont les critiques qui ont noté les réseaux d'amitiés et les salons que forment les conteuses à l'époque. Celles-ci auraient en effet créé une véritable « collectivité créatrice, société où les écrivaines dominent, s'entraident et rendent même hommage à leurs créations en nommant explicitement d'autres contes dans leurs récits merveilleux » (M. Legault, Narrations déviantes, op. cit., p. 187). Sur cet aspect du collectif féminin des conteuses, voir notamment l'œuvre monumentale de Linda Timmermans, L'accès des femmes à la culture sous l'Ancien Régime, 2005, p. 215-224, et celle de Patricia Hannon, Fabulous Identities. Women's Fairy Tales in SeventeenthCentury France, 1998, p. 164-178. Hannon suggère la possibilité de concevoir les contes de fées émanant des salons de cette période comme une « mise-en-scène du corps symbolique des salonnières. [...] les femmes de salon ont construit un Moi symbolique à partir d'un ensemble de contes qui servaient à glorifier les femmes auteures » (« mise-en-scene of the symbolic body of the salonnières. [...] salon women construct a symbolic self through a body of tales that glorify women writers », p. 158).

5 Expression identifiée dans la correspondance de Madame de Sévigné à sa fille : Charlotte Trinquet, « Happily Ever After? Not so Easily! Seventeenth-Century Fairy Tales and their Unconventional Endings », Papers on French Seventeenth-Century Literature, vol. XXXVII, $\mathrm{n}^{\circ}$ 72, 2010, p. 45-54 (p. 48, note 4). " Mitonner » aurait été l'art de raconter des histoires pour plaire aux femmes de la cour. Trinquet cite ici Lewis Seifert, Fairy Tales, Sexuality, and Gender in France, 1996, p. 230, note 25.

6 Patricia Clancy, «The Literary Conte des Fées: A Tale of Survival and Revival », Australian Journal of French Studies, vol. XXXVIII, $\mathrm{n}^{\circ}$ 1, 2001, p. 36-53 : « Perrault et les femmes auteures qui ont établi le conte de fées dans les cercles aristocratiques en France vers les années 1690 ont mis l'emphase sur les bonnes manières, les mœurs, l'art de la conversation et la sensibilité, qualités qui devenaient alors signe de civilité en France » (p. 41). Marcelle Maistre Welch, «La Femme, le mariage et l'amour dans les contes de fées mondains du XVII siècle français », Papers on French Seventeenth-Centure Literature, vol. XX, nº18, 1983, p. 47-58 (p. 47), P. Hannon, Fabulous Identities, op. cit., p. 124 et L. Seifert, Fairy Tales, Sexuality, and Gender, op. cit., p. 68, soulignent également l'importance d'un raffinement culturel et social chez les conteuses.
} 
fées au Grand Siècle va demeurer un fait constant dans l'élaboration de son message central. C'est donc sous la forme de guide ou conseil moral offert aux lectrices que le mentorat va souvent se manifester dans le conte. À cet égard, la fameuse moralité qui chez Charles Perrault s'installe souvent avec beaucoup d'autorité à la conclusion de ses contes atteste en elle-même du caractère instructif du genre merveilleux à l'époque. Prenons Barbe Bleue, publié en 1697, à titre d'exemple. Ici, on s'en souviendra, l'héroïne transgresse l'interdit de son époux et découvre avec horreur, en pénétrant dans une salle dont l'accès lui avait été expressément défendue, une série de cadavres sanglants de femmes, toutes des épouses précédentes de Barbe Bleue qui se sont elles aussi malheureusement hasardées dans l'espace du lieu interdit. Ayant désobéi à l'époux et découvert son secret barbare, l'héroïne est menacée à son tour d'une mort brutale mais elle finira par être sauvée in extremis grâce à l'arrivée de ses frères qui transpercent avec leur épée le corps de Barbe Bleue. À la fin, la « Moralité » de Perrault insiste sur le danger qu'engendre la curiosité féminine, qui ne produit d'ailleurs qu'un plaisir éphémère :

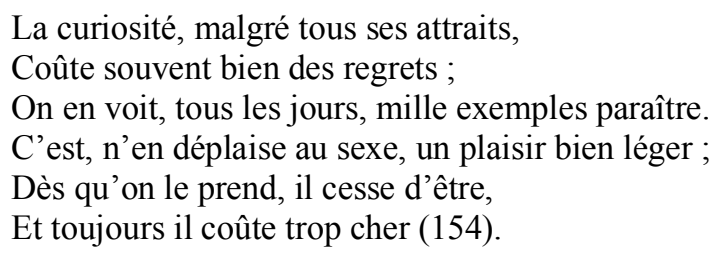

Pour la lectrice, à qui bien sûr s'adresse cette morale condamnatrice, l'avertissement de l'académicien ne saurait être plus clair. Enfin, quoiqu'une « Autre moralité » vienne tant soit peu adoucir le ton didactique de la première en évoquant avec nostalgie moqueuse le «temps passé » du récit de Barbe Bleue ${ }^{7}$, et que la fin du conte libère l'héroïne d'un destin macabre et la laisse même financièrement indépendante à la mort de son mari, la lectrice peut difficilement oublier la vision horrifiante des corps-cadavres et le sort tragique des autres épouses de Barbe Bleue.

$\mathrm{Si}$, dans son conte et par l'entremise de sa Moralité, le mentorat de Perrault se positionne nettement comme une voix instructive, voire même formatrice du sexe féminin, jusqu'où va ce geste de mentorat chez les conteuses et quelles formes prend-il pour guider son public féminin tout en le séduisant par son cadre merveilleux ? Car, pris d'assaut par la plume des auteures féminins, à la fin du dix-septième siècle le conte de fées et son territoire du merveilleux peuvent

\footnotetext{
${ }^{7}$ Sur l'ambiguïté des moralités dans les contes de Perrault, voir L. Seifert, Fairy Tales, Sexuality, and Gender, op. cit., p. 51-58.
} 
permettre aux conteuses et lectrices une certaine échappatoire face aux contraintes imposées par les sphères domestiques et sociales de l'époque, contraintes qui, comme l'ont déjà noté entre autres Lewis Seifert et Patricia Hannon ${ }^{8}$, semblent s'intensifier dans les dernières années du règne de Louis XIV, en partie sous l'influence et l'austérité morales de Madame de Maintenon. Comme s'il adhérait à cette tentative de fin de siècle d'encourager la sobriété morale, la retenue, et la bienséance féminine, comme c'est le cas chez Perrault, le conte féminin va lui aussi servir d'avertissement et donc, forcément, de guide moral à la lectrice. À cet égard, la récurrence des thématiques de l'éducation intellectuelle (fortement louée) et de l'émancipation sexuelle (on s'en doute, toujours désapprouvée) qui sont souvent au cœur de l'intrigue dans le conte de fées féminin ont déjà fait l'objet de maintes enquêtes critiques. ${ }^{9}$ À titre d'exemple de contes féminins qui traitent des dangers de l'émancipation sexuelle, on se souviendra brièvement de La Chatte Blanche et de Persinette dans lesquels les héroïnes de Marie-Catherine d'Aulnoy et de CharlotteRose de Caumont de La Force sont toutes deux punies pour avoir transgressé un interdit sexuel. Dans les deux contes, le sort des princesses est en partie déterminé par le don singulier que font leurs parents. C'est en effet un des parents qui donne sa nouveau-née aux fées en échange pour l'immense plaisir et la satisfaction physiques qu'apportent à la mère enceinte les fruits (dans $L a$ Chatte Blanche) et le persil (dans Persinette) des jardins des fées. Une fois remises entre les mains des fées, chacune des princesses est gardée dans une magnifique tour, apparemment infranchissable. Car les fées veulent avant tout les protéger du monde externe, prévenir leurs fatales destinées et faire leur éducation. Mais, malgré leurs bonnes intentions et toutes leurs précautions, dans La Chatte Blanche la princesse finit par devenir lasse d'avoir pour uniques compagnons un perroquet et un chien et se donne ainsi trop rapidement au premier chevalier qui s'offre un jour à ses yeux. Résultat : les amoureux sont découverts et l'amant se fait dévorer par le dragon des fées, tandis que la jeune princesse est transformée en une chatte blanche. Elle reste ainsi prisonnière de son corps animal pendant de longues années, en attendant qu'un prince identique à son amant lui coupe la queue, afin qu'elle puisse retrouver son identité humaine. Chez La Force, la princesse Persinette est séduite elle aussi par le premier prince qui parvient à s'introduire dans sa tour. Elle consent sur le champ à l'épouser «sans savoir presque ce qu'elle faisait » (335), et devient bientôt enceinte. Comme punition, elle est chassée par les fées, tandis

\footnotetext{
${ }^{8}$ L. Seifert, Fairy Tales, Sexuality, and Gender, op. cit., p. 6 ; P. Hannon, Fabulous Identities, op. cit., p. 33.

${ }^{9}$ Voir notamment les articles de Marcelle Maistre Welch, Michèle Farrell, Kathryn Hoffmann et Carolyn Vellenga.
} 
que son mari devient aveugle. Condamnée à errer seule avec ses enfants, après plusieurs années de séparation, Persinette et son mari se retrouvent par hasard un jour; la fée, attendrie par le spectacle de la fidélité et de la constance des époux, redonne la vue au mari. Pour la lectrice du Grand Siècle, ces deux scénarios démontrent assez clairement les dangers associés à l'émancipation et la liberté sexuelles féminines. Si en fin de compte tout est bien qui finit bien, la lectrice, quant à elle, ne peut oublier que les deux héroïnes souffrent toutefois pendant de longues années précisément à cause de leur transgression sexuelle. Ces conteuses offrent ainsi une leçon sans équivoque. Dans les deux cas, les auteures se posent comme mentors et guides morales en insistant sur la nécessité de faire preuve d'une certaine austérité sexuelle féminine. Leurs contes servent ainsi d'avertissement aux lectrices.

C'est pour son rôle souvent à la fois formateur et instructif que le conte de fées se prête particulièrement bien à une étude sur le mentorat. Car, en plus de vouloir séduire avec son décor magique, son enjeu est souvent d'éduquer. Cependant, le mentorat dans le conte n'y prend pas toujours une allure morale austère. En effet, malgré l'avertissement assez transparent qu'offrent La Chatte Blanche et Persinette, certaines conteuses utilisent le mentorat féminin pour en faire un outil qui, loin d'affecter un goût pour la retenue morale, vient au contraire encourager la lectrice de l'Ancien Régime vers une conduite moins moralement prescrite et qui va même, dans certains cas, à l'encontre des attentes sociales de leur époque. J'espère démontrer quelques-uns des moyens par lesquels, grâce à leur mentorat, ces conteuses promeuvent la liberté féminine et réussissent parfois même à dissimuler une série de transgressions sous le masque de leçons de morale, par des maximes, ou encore dans la conclusion de leurs intrigues. Malgré la présence de la moralité finale et de leur devoir littéraire de «plaire pour instruire », les contes de Catherine Bernard, Marie-Jeanne L'Héritier, et Rose-Charlotte de La Force utilisent le mentorat féminin soit pour dénoncer les hommes, pour stimuler la création littéraire féminine, ou encore pour encourager une existence féminine plus libre. Dans chaque cas, il s'agit pour la conteuse de guider la lectrice par un mentorat, qui trahit toujours un geste complice envers celle-ci, à explorer un cheminement à la fois moral et social auquel on s'attend peu.

Prenons d'abord le Riquet à la Houppe de la romancière et conteuse Catherine Bernard, publié en 1696. Le récit se situe à Grenade et raconte l'histoire amoureuse de l'héroïne espagnole Mama qui a la mauvaise fortune d'être née sans aucune trace d'intelligence : 
[Mama] née avec tous les traits qui font la beauté, était si stupide que la beauté même ne servait qu'à la rendre désagréable. Ses actions n'avaient rien de ce qui fait la grâce ; sa taille, quoique déliée, était lourde, parce qu'il manquait une âme à son corps ${ }^{10}$.

Sa stupidité est telle que Mama «n'avait pas assez d'esprit pour savoir qu'elle n'en avait point $»^{11}$, nous informe le texte, non sans humour. Lors d'une promenade, elle fait un jour la rencontre d' « un homme, assez hideux pour être un monstre $»^{12}$, sorti de la terre pour lui faire une offre qu'elle ne pourra pas refuser. En effet, afin de remédier à son triste sort, Mama accepte d'épouser ce monstre des lieux souterrains, l'affreux bossu Riquet, qui lui a promis en échange de la doter d'intelligence et de bel esprit. Séduite par la pensée d'avoir de l'esprit, Mama promet d'épouser Riquet dans un an. Aussi, le monstre lui enseigne-t-il sur le coup à prononcer les paroles magiques qui la rendront intelligente et qui lui inspireront de l'amour pour lui. Il est important de noter qu'il ne s'agit donc pas ici d'un conte qui dénonce les injustices du mariage forcé, un thème que l'on rencontre couramment chez d'autres conteuses, comme c'est le cas dans L'Enchanteur que nous aborderons plus loin. Notre héroïne, au contraire, est ici en plein contrôle de sa destinée.

Pendant le délai d'un an, Mama, qui jusqu'alors avait été « dédaignée » de tous, son imbécillité la rendant odieuse, fait soudainement tomber les cœurs auprès d'elle : « Les amants vinrent en foule ; Mama ne fut plus solitaire ni au bal ni à la promenade ; elle fit bientôt des infidèles et des jaloux ; il n'était bruit que d'elle et que pour elle ${ }^{13}$. Contrairement aux intentions de Riquet, si les paroles du monstre font naître de l'amour dans le cœur de Mama, c'est toutefois pour un autre homme : le bel Arada. On notera qu'à ce point dans son récit Bernard prend bien soin d'exonérer son héroïne en soulignant par une maxime la fatalité des forces de l'amour et de la nature sur Mama : «Mais défendre d'aimer à une jeune et jolie personne, ce serait défendre à un arbre de porter des feuilles au mois de mai ${ }^{14}$. Cette première justification, qui s'insère dans le texte comme un geste sympathique envers l'écart de conduire de l'héroïne, va s'avérer cruciale pour mieux comprendre le combat difficile qui commence à se dessiner chez Mama entre amour et intelligence. En effet, désormais pourvue d'intelligence, au fur et à mesure que l'échéance des

\footnotetext{
${ }^{10}$ Catherine Bernard, Riquet à la Houppe, 1696, dans Raymonde Robert (éd.), Contes. Bibliothèque des Génies et des Fées, 2005, p. 287-292 (p. 287).

${ }^{11}$ Ibid.

12 Ibid.

${ }^{13}$ Ibid., p. 288.

${ }^{14} \mathrm{Ibid}$.
} 
douze mois approche, l'incertitude de Mama augmente cruellement devant son impasse : en épousant Riquet elle devra renoncer à Arada, mais en refusant de l'épouser elle redeviendra imbécile et perdra par conséquent l'amour de son amant. C'est finalement «l'idée de perdre son amant par le mépris qu'il aurait pour elle » qui la pousse à accepter d'épouser Riquet ${ }^{15}$. Mais, dorénavant liée à « un époux odieux », l'étendue du malheur de Mama ne fait que redoubler à ses yeux :

[Riquet] l'épousa et l'esprit de Mama augmenta encore par ce mariage, mais son malheur augmenta à proportion de son esprit; elle fut effrayée de s'être donnée à un monstre et à tous moments elle ne comprenait pas qu'elle pût passer encore un moment avec lui1' ${ }^{16}$.

L'image du couple marié que peint Bernard à partir de ce moment est des plus sombre. On n'y retrouve que haine, aversion, et isolation, sentiments éprouvés tant par Mama que par Riquet mais qui, on s'en doute, sont intensifiés chez la pauvre héroïne pour qui son mari reste « un monstre $»^{17}$. Malheureuse et forcée de demeurer souvent seule dans le royaume souterrain du gnome, où tous ceux qui l'entourent sont difformes comme Riquet, l'esprit de Mama se tourne alors vers Arada qu'elle veut convaincre, malgré son mariage, de sa passion fidèle et qui, elle espère, pourra l'aider à supporter son malheur. Elle trouve ainsi les moyens de contacter son amant. De nouveau, Bernard interrompt soudainement la narration de son récit par une seconde maxime qui vient encore une fois justifier la conduite de Mama : «Il n'est-il rien d'impossible à une femme d'esprit qui aime ${ }^{18}$. Tandis qu'on pourrait déduire ici, comme le fait Seifert, que Bernard tisse un lien étroit entre l'acquisition d'une intelligence et le vice chez la femme, l'enclos oppressif et l'environnement monstrueux où l'héroïne est coincée, symbolisant bien sûr les dangers de l'institution du mariage pour la femme, nécessitent des mesures extraordinaires (« rien d'impossible ») ${ }^{19}$. Comme on l'a vu auparavant, Bernard défend (cette fois par la peinture d'un mariage des plus oppressant) le geste trompeur de son héroïne. Mais elle va plus loin : sa maxime se pose aussi, en quelque sorte, comme un conseil à la lectrice de voir dans le scénario de Bernard une issue possible (la déception) dans le malheur domestique. Car, rappelons-le, toute la tragédie du conte de Bernard repose sur le choix de Mama d'épouser Riquet, un monstre dont

\footnotetext{
${ }^{15}$ Ibid.

${ }^{16}$ Ibid., p. 289-290.

${ }^{17}$ Ibid., p. 290.

${ }^{18}$ Ibid.

${ }^{19}$ Ibid.
} 
l'aspect hideux est souligné sans relâche dans le conte. Si le texte joue sur la vieille dichotomie traditionnelle entre intelligence (domaine du masculin, transmis ici par Riquet) et amour (domaine du féminin), le mariage demeure dans le texte de Bernard une union regrettable, voire même suffocante, et le mari, quant à lui, toujours un monstre. Dans ce contexte, la maxime de Bernard suggère une solution possible au malheur domestique, c'est-à-dire l'adultère. Son geste de mentorat féminin pour contrer le malheur matrimonial de l'héroïne (et par extension des lectrices) est certes exceptionnel.

Maman étant coincée dans ce cauchemar domestique, son amant n'hésite pas à venir la rejoindre clandestinement dans les lieux souterrains du royaume de Riquet. Cependant, l'idylle amoureuse secrète ne dure qu'un temps. Le monstre-mari, remarquant un changement d'humeur chez Mama, devine la présence de l'amant et, par conséquent, la déception de sa femme : «quand je vous ai donné de l'esprit, je prétendais en jouir; vous en avez fait usage contre moi $»^{20}$. L'intention du maitre-époux ne saurait être plus clair : Riquet se veut l'unique bénéficiaire de l'esprit de Mama, et pour punir sa femme, il lui redonne une part de sa stupidité :

vous aurez de l'esprit la nuit, je ne veux point d'une femme stupide ; mais vous le serez le jour pour qui il vous plaira. Mama dans ce moment sentit une pesanteur d'esprit que bientôt elle ne sentit même plus. La nuit, ses idées se réveillèrent; elle fit réflexion sur son malheur; elle pleura, et ne put se résoudre à se consoler, ni à chercher les expédients que ses lumières lui pouvaient fournir ${ }^{21}$.

Mama sera dorénavant intelligente la nuit, pour lui et pour son plaisir, et bête le jour, pour Arada. La vengeance du mari afflige Mama mais ne peut altérer la nécessité absolue qu'elle ressent de «s'éloigner de l'objet de son courroux $»^{22}$. Ainsi, possédant toujours de l'esprit la nuit, quand Riquet s'est endormi auprès d'elle la nuit suivante Mama lui glisse une herbe somnifère sous le nez (symbolisant son pouvoir phallique), pour l'endormir profondément. Désormais, elle peut éviter tout contact physique avec son monstrueux mari la nuit et devient libre d'aller rejoindre l'objet de sa passion, Arada. Le jour venu, Mama réveille le monstre et passe ensuite le reste du temps à dormir.

Après plusieurs nuits, le stratagème est éventuellement découvert quand le domestique de Riquet, inquiété par le ronflement de son maître, lui vient en aide en retirant l'herbe. Le mari surprend aussitôt les amoureux et, comme punition, Riquet transforme Arada en une copie exacte

${ }^{20}$ Ibid., p. 291.

${ }^{21}$ Ibid.

${ }^{22}$ Ibid. 
du monstre. Mama ne pourra dorénavant échapper ni à la monstruosité du mari, ni à celle de l'amant. Son sort est donc doublement tragique. On pourrait très bien lire dans le conte de Bernard un avertissement sévère (suivant le geste de mentorat féminin austère qui s'inscrit dans La Chatte Blanche et Persinette) contre l'infidélité féminine ou encore y voir un message inquiétant à l'égard d'une trop grande émancipation intellectuelle de la femme. Mais les deux dernières phrases du conte viennent bouleverser cette conclusion trop hâtive : "mais peut-être qu'elle n'y perdit guère : les amants à la longue deviennent des maris $»^{23}$. Bernard termine son conte non pas avec une moralité conventionnelle, mais avec une dernière maxime, cette fois fort pessimiste et qui sert d'avertissement général contre les hommes : qu'il s'agisse de maris ou d'amants, les hommes deviennent tous avec le temps des maris, c'est-à-dire, monstrueux. Le conseil de Bernard dans cette maxime qui sert de clôture finale au récit ne saurait être plus explicite pour la lectrice. Mama, comme son nom (dérivatif de maman) le suggère dès le début du conte ${ }^{24}$, ne peut à la longue et malgré ses nombreux efforts échapper à l'étau de la domesticité. Dans son conte, Bernard utilise ainsi la maxime comme une forme oblique de mentorat féminin et ce dans deux buts : le premier, pour justifier la conduite de son héroïne adultère, conduite qui, sans cette justification narrative, serait moralement fort problématique. Par ailleurs, cette justification complice a pour effet d'encourager la lectrice à y voir elle-même une échappatoire au malheur domestique. Le second, pour avertir la lectrice de l'ultime danger que (malgré les plaisirs de l'amour) représentent pour elles les hommes, ainsi que des malheurs certains qui découlent de l'institution du mariage.

En guise de conclusion au conte de Bernard, on notera l'arrivée d'un deuxième Riquet à la Houppe dans la production des contes de fées à l'époque. En effet, étant donné que le Riquet de Bernard tisse un tableau fort sombre du mari, un être à la fois dominant et monstrueux, il n'est pas étonnant que Perrault publie sa propre version du Riquet un an plus tard, soit en 1697. Dans le Riquet à la Houppe de Perrault, deux changements sont d'importance. Le premier: la perspective a entièrement changé. C'est dorénavant l'histoire du pauvre Riquet et non celle de Mama dont il est question. Dès lors, on s'étonne peu que la monstruosité de Riquet y soit

\footnotetext{
${ }^{23}$ Ibid., p. 85.

${ }^{24} \mathrm{Au}$ sujet du prénom de Mama, j'adopte ici la même hypothèse que Seifert pour qui son nom « is the means by which the tale denounces the heroine's gradual but no less certain enclosure in maternal domesticity » (211); (est le moyen par lequel le conte dénonce la progression graduelle mais pas moins certaine de l'héroïne vers l'enceinte maternelle domestique).
} 
beaucoup moins soulignée. Mais s'il est toujours « laid» et «mal fait $»^{25}$, le héros de Perrault est, en revanche, plus sympathique que menaçant et démontre aussi plus d'esprit que celui de Bernard. Le deuxième : Mama ne ressent pas la même horreur devant Riquet qu'on remarque chez l'héroïne de Bernard. En fait, elle finit par voir en Riquet «l'homme du monde le plus beau, le mieux fait et le plus aimable qu'elle eût jamais vu $»^{26}$. En plus de ces deux transformations majeures qui viennent, en quelque sorte, censurer le message de Bernard sur les hommes et le mariage, la moralité finale de Perrault dénonce de façon oblique le Riquet à la Houppe de Bernard :

\footnotetext{
Ce que l'on voit dans cet écrit, Est moins un conte en l'air que la vérité même ; Tout est beau dans ce que l'on aime,

Tout ce qu'on aime a de l'esprit ${ }^{27}$.
}

Il semble bien que pour Perrault, le «conte en l'air » de Bernard, mettant en scène un époux aussi hideux que menaçant et offrant un avertissement final contre les hommes, se devait d'être refait pour permettre au statu quo de l'économie sociale et sexuelle d'être maintenu. Le Riquet de Perrault remet ainsi les choses à leurs places.

Contrairement au conte de Bernard où le mentorat prend la forme d'un geste complice qui avise explicitement la lectrice sur les dangers des hommes et du mariage grâce à sa maxime qui clôt le Riquet à la Houppe, dans le conte de Marie-Jeanne L'Héritier, L'Adroite princesse ou les aventures de Finette (1696), le mentorat féminin s'effectue dès les premières lignes. Mais le mentorat ici revêt encore plus les couleurs d'un échange entre complices. Chez L'Héritier, en effet, l'espace du conte est marqué d'un mentorat féminin dissimulé dans un partage intime entre amies et dans un appel à la création littéraire féminine. L'Adroite princesse est l'histoire de trois sœurs dont la vertu est mise à l'épreuve par un prince séduisant et malicieux. Tandis que les deux sœurs de l'héroïne tombent victimes du méchant prince (Nonchalante et Babillarde se font tour à tour séduire, deviennent enceintes, et finissent enfin par mourir), la sage Finette échappe, elle, aux avances trompeuses du prince et finit même par le tuer. Les aventures de la prudente et courageuse héroïne Finette soulignent bien sûr un des thèmes chers au conte féminin, soit l'importance de la vertu et de la gloire féminines. En cela, le récit de L'Hériter perpétue la

${ }^{25}$ Charles Perrault, Riquet à la Houppe, dans Contes, éd. J. P. Collinet, 1981, p. 181.

${ }^{26}$ Ibid., p. 187.

${ }^{27}$ Ibid., p. 188 ; c'est moi qui souligne.

https://journals.uvic.ca/index.php/sator/index 
tradition du mentorat dans le conte, c'est-à-dire qu'il sert de guide moral à la lectrice. Mais les aventures de Finette sont aussi encadrées par un long prologue et un épilogue dont L'Héritier se sert pour divertir mais aussi pour séduire son amie, la romancière Henriette-Julie de Murat, vers l'écriture du genre merveilleux. Le mentorat féminin chez l'Héritier se pose ainsi comme un geste intime qui a pour but ultime d'encourager l'amie à se joindre à la vogue de la production littéraire du conte de fées. Le caractère intime du mentorat de l'auteure se révèle dès les premières lignes. Dans son prologue, en effet, L'Héritier s'adresse directement et avec galanterie à la comtesse de Murat à qui elle dédie son conte :

Vous faites les plus jolies nouvelles du monde en vers ; mais en vers aussi doux que naturels. Je voudrais bien, charmante comtesse, vous en dire une à mon tour [...] en un mot, un récit sans façon et comme on parle : je ne cherche que quelque moralité. Mon historiette en fournit assez, et par là elle pourra vous être agréable ${ }^{28}$.

L'Héritier annonce déjà que la moralité (avec son message instructif) tiendra une place d'importance dans son conte, mais que cette moralité plaira à son amie. Le double objectif littéraire de "plaire et instruire », suivant l'esthétique de l'époque, est donc explicitement énoncé. Le prologue se poursuit avec un conseil qui reflète le message central du conte et selon lequel les femmes devraient éviter l'oisiveté et toujours s'armer de défiance vis-à-vis des hommes. Dans cette lutte, c'est l'intelligence féminine qui fournira l'outil de combat par excellence :

Non l'amour ne triomphe guère

Que des cœurs qui n'ont point d'affaires.

Vous, qui craignez que d'un adroit vainqueur

Votre raison ne devienne la dupe,

Beautés, si vous voulez conserver votre cœur,

Il faut que votre esprit s'occupe ${ }^{29}$.

On notera que loin de s'adresser uniquement à Murat, son conseil s'étend ici à toutes les lectrices («beautés »). L’Héritier poursuit sa leçon en soulignant le besoin d'être méfiantes face aux hommes :

Mais si, malgré vos soins, votre sort est d'aimer,

\footnotetext{
${ }^{28}$ Marie-Jeanne de Villandon L'Héritier, L'Adroite princesse ou les aventures de Finettte, 1696, dans Raymonde Robert (éd.), Contes. Bibliothèque des Génies et des Fées, 2005, p. 93-114 (p. 93).

${ }^{29}$ Ibid.
} 


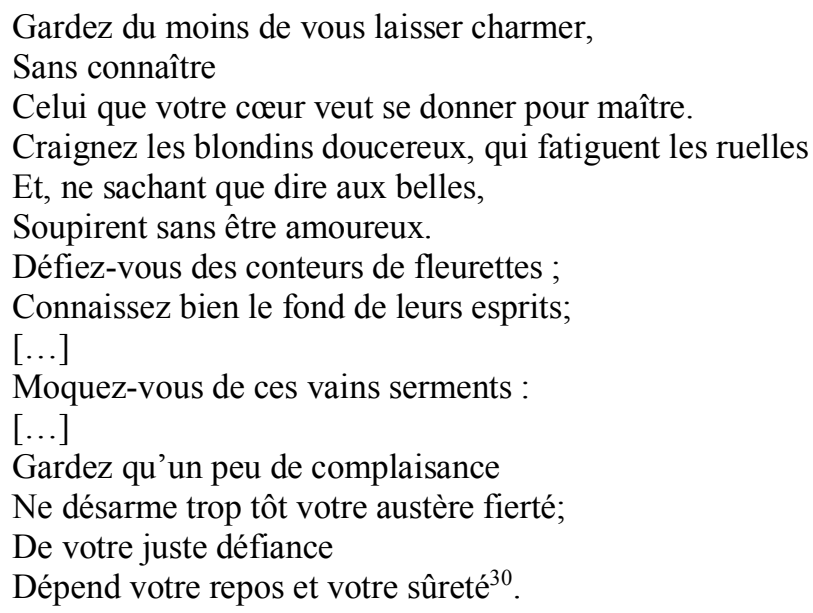

Dès son prologue, L'Héritier met donc l'emphase sur le mentorat féminin. Elle prend au sérieux son rôle de guide moral sur la lectrice et sur Murat. En fait, tout au long du conte, L'Héritier continue sa conversation avec son amie. En effet, en plus d'incorporer son amie au prologue et à l'épilogue, l'auteure interrompt sa narration à quatre reprises pour s'adresser, non sans galanterie, à Murat, comme dans cet exemple : "Vous qui êtes si savante dans toutes sortes d'antiquités, je ne doute pas, Comtesse charmante, que vous n'ayez cent fois entendu parler du merveilleux pouvoir des fées $»^{31}$. Ces interpellations sont importantes parce qu'elles vont mener à la tentative de L'Héritier d'inciter Murat à écrire des contes de fées. Ainsi, après le récit de Finette et de ses deux imprudentes sœurs, L'Héritier conclut son conte par une moralité qui sert d'invitation tout aussi directe que séduisante à Murat :

\footnotetext{
Mais ces fables plairont jusqu'aux plus grands esprits,

Si vous voulez, belle Comtesse,

Par vos heureux talents, orner de tels récits.

L'antique Gaule vous en presse,

Daignez donc mettre dans leurs jours

Les contes ingénus, quoique remplis d'adresse

Qu'on inventés les troubadours ${ }^{32}$.
}

L'Héritier fait donc preuve d'un double mentorat dans son conte. D'une part son récit sert d'avertissement général aux femmes sur les dangers qu'entrainent l'oisiveté d'esprit et le manque de défiance vis-à-vis des hommes. D'autre part, son prologue et son épilogue qui s'adressent principalement à Murat ont pour objectif d'inciter son amie à l'écriture du

${ }^{30}$ Ibid., p. 93-94.

${ }^{31}$ Ibid., p. 97-98.

${ }^{32}$ Ibid., p. 114.

https://journals.uvic.ca/index.php/sator/index 
merveilleux. Il s'agit en quelques sortes d'un jeu séducteur, où le mentorat de l'auteur sert d'exemple littéraire à Murat. On note aussi que L'Héritier souligne ici la popularité du conte de fées auprès de l'élite mondaine («grands esprits »). Enfin le mentorat de L'Héritier et son appel à l'écriture du genre merveilleux firent effet sur Murat, qui publiera entre 1697 et 1708 ses propres contes de fées. Mais certains de ses contes, comme Anguillette (1698) et Peine Perdue (1708) se rapprochent davantage du réel que du merveilleux; de fait, la magie, l'amour et le héros féminin y ont perdu beaucoup d'éclats et de promesses. Résultat: le cheminement du personnage féminin ainsi que le destin final sont des plus tragiques pour l'héroïne. Chez Murat, l'élan optimiste des conteuses qui l'ont inspirée s'est nettement transformé. Le merveilleux y est à peine reconnaissable.

D'un jeu de séduction menant l'amie à l'écriture du merveilleux chez L'Héritier, le mentorat féminin sous la plume de La Force passe à l'approbation d'une conduite féminine moralement équivoque. La moralité finale de son conte L'Enchanteur (1697) encourage en effet la lectrice à faire son propre cheminement vers le bonheur, quelle que soit sa conduite. L'Enchanteur s'inscrit ainsi comme un conte des plus libérateur pour la lectrice. L'auteure ne cache pas dans son «Avis » aux lecteurs, que loin d'être original, son conte prend sa source d'un « ancien livre gothique, nommé Perseval $»^{33}$. Jacques Barchilon et Raymonde Robert ont tous deux noté qu'il ne s'agit pas du Perceval de Chrétien de Troyes, mais d'une continuation de cette œuvre, publiée par un auteur anonyme vers $1530^{34}$. La Force, selon son propre aveu, n'y aurait retranché que quelques détails qui, explique-t-elle, «n'étaient pas suivant nos mœurs $»^{35}$. Le récit anonyme contenait en effet certains épisodes plutôt choquants, tels que les accouplements de l'Enchanteur avec des animaux, et qui nécessitaient forcément d'être remaniés ${ }^{36}$. Malgré les changements qu'elle affirme avoir apportés à la version originale, le conte de La Force, qui met en scène un amour adultère, demeure toutefois un des contes les plus violent mais en même temps les plus érotique du répertoire merveilleux de l'époque.

\footnotetext{
${ }^{33}$ Rose-Charlotte de Caumont de La Force, L'Enchanteur, 1697, dans R. Robert (éd.), Contes. Bibliothèque des Génies et des Fées, op. cit., p. 339-355 (p. 339)

${ }^{34}$ Jacques Barchilon, "L'Enchanteur de Mlle de La Force », Merveilles et contes, 1988, vol. 2, n 1, p. 47-60 (p. 47) ; R, Robert, Contes, op. cit., p. 299.

${ }^{35}$ R.-C. de Caumont de La Force, L'Enchanteur, éd. cit., p. 339.

${ }^{36}$ Dans son édition critique, Raymonde Robert explique que dans le texte de 1530, l'Enchanteur était condamné à coucher pendant trois nuits de suite avec trois animaux différents : une levrette, une truie et une jument. De ces accouplements sont nés trois « frères » de Carados, autre héros du conte (L'Enchanteur, p. 300).
} 
Sans trop insister sur les détails complexes de ce conte, on notera toutefois que c'est l'histoire de l'Enchanteur qui tombe amoureux d'Isène la Belle, la nièce d'un «bon roi », qui vient d'être promise en mariage à un monarque d'un royaume voisin. Le soir des noces d'Isène, l'Enchanteur, «qui fut très fâché de voir qu'elle allait être à un autre $»^{37}$, met dans le lit du roi, grâce à sa magie, une esclave que ce dernier prend pour son épouse, Isène. Enfin libre de déclarer son amour à la belle Isène, il lui avoue du même coup comment il vient de tromper le roi :

Il dit à la reine les plus belles choses du monde ; elle y répondit si bien qu'il se crut heureux, et il lui avoua qu'il avait mis, dans le lit du roi, une esclave qu'il prendrait pour elle. Isène en rit, et passa la nuit à se moquer de son mari, et le jour étant venu, elle parut comme si de rien n'était.

Le roi, fort charmé de la bonne fortune qu'il avait eue, se trouvait le plus content de tous les hommes, mais $l^{\prime} E n c h a n t e u r$ était le plus amoureux et le plus satisfait ${ }^{38}$.

On remarque que La Force insiste d'une part sur le plaisir et la satisfaction physiques que ressent l'amant après avoir passé la nuit auprès d'Isène, et d'autre part sur le caractère impudique de la jeune femme, qui ne se sent nullement coupable de ses actions et qui dissimule même parfaitement bien toute marque de sa conduite adultère le lendemain. Mais, ce qui est encore plus surprenant est le manque total de condamnation morale dans la narration de La Force à l'égard du couple adultère. En fait, on comprend dès le début du conte que les forces de l'amour ont le dessus sur la puissance du merveilleux dans ce récit magique. En effet, avant même de rencontrer son amant le soir de ses noces, l'héroïne quitte son lit nuptial, poussée «par une puissance secrète " vers un autre cabinet où survient l'Enchanteur ${ }^{39}$. Puis, lors de cette première rencontre avec l'Enchanteur dans laquelle il lui déclare promptement sa passion, le texte précise qu'Isène «sentit une si grande inclination pour lui, que toute la magie ne peut former rien de semblable, s'il n'est pris dans un sentiment naturel $»^{40}$. Isène est en quelque sorte exonérée, étant victime des forces de l'amour et de la nature qui, même dans le cadre merveilleux du conte, l'emportent de toute évidence sur le pouvoir de la magie. Le récit de La Force ne condamne ainsi à aucun moment les amants dans les descriptions de leurs rendez-vous amoureux :

[...] il donna cent marques d'amour à Isène la Belle, où personne ne prit garde ; ils se regardaient à la dérobée, s'ils dansaient ensemble, ils se serraient la main, ils buvaient à table dans le même verre, rien n'est comparable à la félicité des commencements d'amour.

\footnotetext{
${ }^{37}$ R.-C. de Caumont de La Force, L'Enchanteur, éd. cit., p. 339.

${ }^{38}$ Ibid., p. 340.

${ }^{39}$ Ibid., p. 339.

${ }^{40}$ Ibid., p. 340.
} 
La seconde nuit, l'Enchanteur fut encore avec la reine, et il mit son esclave dans le lit du roi. La journée se passa en ces témoignages d'amour qui, bien que donnés mystérieusement, ont un charme infini pour les âmes délicates ${ }^{41}$.

Loin de condamner les actions immorales des amants, le texte peint surtout un tableau fort réel de leur relation illicite et insiste même sur l'intensité de leurs émotions.

Le scénario adultère de la nuit de noces se répète de la sorte pendant trois nuits consécutives, jusqu'à ce que le roi conduise Isène dans son royaume. Là, elle donne naissance à un fils (Carados) qui, on s'en doute, est celui de l'Enchanteur. Le conte de La Force débute ainsi avec le thème de l'adultère. Si le thème de l'infidélité n'est pas étranger au genre du merveilleux ${ }^{42}$, l'adultère (surtout commis contre un monarque) y est toutefois fort rare. Un fait encore plus unique est qu'il apparaît avec La Force de manière explicite et sans jugement moral découlant de la narration. En fait, si l'adultère d'Isène finit par être découvert, elle en est punie par un acte qui s'impose surtout comme étant contre nature puisque la vengeance vient principalement de son fils qui a tout exposé au roi :

On envoya chercher Isène la Belle qui se pâma en entendant dire la vérité, et qui n'en parue que trop convaincue; elle ne s'amusa pas à la nier, mais sa plus grande douleur était de se voir accuser et convaincue par son propre fils ${ }^{43}$.

Sans sembler vouloir insister sur le passé immoral d'Isène, le texte souligne plutôt le geste inquiétant et anormal du fils envers sa mère. Car c'est Carados qui conseille le roi (qu'il regarde comme son père) pour l'aider à concevoir une punition. Pour le fils, « un si grand mal » nécessite forcément « une vengeance d'éclat » ${ }^{44}$. Sous les conseils de Carados, le roi ordonne de faire bâtir une tour infranchissable pour y emprisonner sa femme. L'enfermement brutal d'Isène pourrait servir d'avertissement sur la conduite immorale de l'héroïne, si elle seule était punie dans le conte. Mais La Force tisse des scènes punitives et violentes pour les quatre personnages principaux. Après Isène, l'Enchanteur (qu'on surprend à divertir la reine dans la tour) devient aussi la victime de Carados qui veut «le tourmenter d'une peine ignominieuse ${ }^{45}$. À l'aide

\footnotetext{
${ }^{41}$ Ibid.

${ }^{42} \mathrm{C}^{\prime}$ est le cas dans le conte de L'Héritier où les deux sœurs de Finette sont séduites simultanément par le méchant prince qui promet un amour éternel à chacune d'elles. Comme on l'a vu, le prologue souligne bien d'ailleurs la nécessité de se méfier de la passion masculine, ou du moins d'en éprouver la constance.

${ }^{43}$ R.-C. de Caumont de La Force, L'Enchanteur, éd. cit., p. 345.

${ }^{44}$ Ibid.

${ }^{45}$ Ibid.
}

https://journals.uvic.ca/index.php/sator/index 
d'une fée, il place une esclave à la ressemblance d'Isène dans le lit de l'Enchanteur pendant trois nuits pour lui faire ressentir « une honte éternelle de son infidélité » ${ }^{46}$. Enfin, le fils contre-nature « qui ne sen[t] nul remords du traitement qu'il fai[t] à sa mère » finit par subir la vengeance maternelle : une morsure douloureuse d'une serpente qui s'attache à son bras défigure Carados au point où personne ne le reconnaît, telle est l'étendue de son « horrible changement ${ }^{47}$. Mais Isène s'adoucit et révèle l'unique moyen de libérer son fils de sa peine : seule une «pucelle qui fut aussi constante que belle », en offrant son corps à la serpente peut le sauver ${ }^{48}$. C'est ici que la jeune et angélique Adelis, longtemps amoureuse de Carados, leurre la serpente à s'attacher à elle; mais, au moment où son frère devait trancher la serpente avec son épée, il coupe malheureusement aussi le bout du sein d'Adelis. Les personnages principaux, quelle que soit leur conduite, sont donc tous mis à l'épreuve.

Après le pardon royal qu'on donne à Isène et la mort subite et inexpliquée du monarque qui s'apprêtait à la remarier, à la fin du conte l'Enchanteur devient libre d'épouser Isène : « Il demanda Isène la Belle pour sa femme, on la lui accorda. Il était bien juste de récompenser une si longue amour, si ardente et si fidèle. Ces quatre époux vécurent dans un bonheur perpétuel ${ }^{49}$. Comme si tout le conte, et particulièrement sa conclusion, ne rendait pas suffisamment hommage à la passion adultère de l'Enchanteur et d'Isène, la moralité finale de La Force y fait aussi sa part :

\footnotetext{
Par différents chemins on arrive au bonheur, Le vice nous y mène, aussi bien que l'honneur ; Témoin ce que l'on voit en Isène la Belle, Après des tourments mérités, Elle eut mille prospérités ${ }^{50}$.
}

Il est vrai que la moralité souligne « les tourments mérités » d'Isène. Mais, comme on l'a vu, les personnages principaux souffrent tous à part plus ou moins égale, y compris la vertueuse Adélis. Vertus et vices sont donc victimes de semblables châtiments. En fin de compte, La Force ne peint jamais son héroïne Isène comme une mauvaise femme adultère. Les forces de l'amour ayant triomphé dès le début sur Isène et l'Enchanteur, l'auteure lui permet au contraire une fin

\footnotetext{
${ }^{46}$ Ibid., p. 346.

${ }^{47}$ Ibid., p. 345, 351.

${ }^{48}$ Ibid., p. 351. On reconnaît qu'en choisissant une serpente, La Force souligne le pouvoir phallique de la mère.

${ }^{49}$ Ibid., p. 354.

${ }^{50} \mathrm{Ibid}$.
} 
heureuse, auprès de celui qu'elle a toujours aimé. Car rappelons-le, Isène est le prix d'un échange entre hommes dès le début du conte. Si La Force n'insiste jamais sur la perspective d'Isène dans ce mariage arrangé entre monarques et qui prend place au début du récit, la lectrice ne peut oublier que cette union n'est pas basée sur l'amour, ni même sur le choix d'Isène. Dans cette optique, l'adultère d'Isène deviendrait alors tout à fait excusable. Mais La Force fait beaucoup plus. Souvenons-nous que dans sa moralité finale l'auteure guide explicitement la lectrice à suivre librement son propre chemin, quel qu'il soit, mené par le vice ou par la vertu : « Par différents chemins on arrive au bonheur, / Le vice nous y mène, aussi bien que l'honneur ». Adultère ou vertueuse, le «on» et le «nous», pronoms qui s'affichent comme des marques nettement complices entre auteure et lectrices, ne sauraient être autres que féminins chez La Force. Le geste audacieux de l'auteure peut surprendre, surtout après la lecture de Persinette. Mais La Force déclarera quatre ans plus tard dans son autoportrait son goût pour le nonconformisme: «je bannis la contrainte, [...] mon esprit est fort libertin; j'ai l'imagination échauffée » («Mademoiselle» xxiv) ${ }^{51}$. Conforme à son désir d'éliminer toute contrainte, dans son conte L'Enchanteur La Force encourage nettement la lectrice à suivre son propre cheminement moral et sexuel. En ce faisant, elle appose sa propre marque de mentorat féminin sur le conte de fées.

Qu'ils soient séduisants comme le conte de L'Héritier, pessimistes comme le Riquet de Bernard, ou revendicateurs de la liberté sexuelle féminine comme dans L'Enchanteur de La Force, les contes de fées à la fin du Grand Siècle peuvent devenir des lieux d'évasions créatrices qui permettent l'articulation de messages et de conseils souvent obliques sur la conduite féminine. Chez ces conteuses, le mentorat féminin apparaît soit grâce à des maximes, soit par l'entremise de prologue et d'épilogue séducteurs, ou encore dans la moralité finale et libératrice. Dans chaque conte, l'auteure se positionne comme une mentor-complice pour dénoncer les hommes, pour promouvoir la création littéraire du genre merveilleux chez la femme, et enfin pour encourager la lectrice à suivre son propre cheminement moral. Bernard, L'Héritier et La Force ont certes fait preuves de ruses dans leur mentorat féminin qui s'impose en geste transgressif puisqu'il insiste, dans chaque cas et à différent degré, sur la poursuite de la liberté du moi féminin.

${ }^{51}$ R.-C. de Caumont de La Force, « Mademoiselle de La Force peinte par elle-même », Les Jeux de l'esprit ou La Promenade de Conti, 1701 (Paris, 1862, xxiv). Également cité dans M. Legault, Narrations déviantes, op. cit., p. 189. 
Marianne Legault

The University of British Columbia

\section{BIBLIOGRAPHIE}

AULNOY, Catherine, La Chatte Blanche. 1697, dans Le Cabinet des fées, t. 1., Arles, P. Picquier, 1994. [Elisabeth Lemirre (éd.)]

BARCHILON, Jacques, "L'Enchanteur de Mlle de La Force », Merveilles et contes, Wayne State University Press, 1988, vol. 2, no 1, p. 47-60. [http://www.jstor.org/stable/41390239]

BERNARD, Catherine, Riquet à la Houppe, 1696, dans Contes. Bibliothèque des Génies et des Fées, Paris, Champion, 2005, p. 287-292. [Raymonde Robert (éd.)]

CLANCY, Patricia, «The Literary Conte des Fées: A Tale of Survival and Revival », Australian Journal of French Studies, vol. XXXVIII, n 1, 2001, p. 36-53.

FARRELL, Michèle L., "Celebration and Repression of Feminine Desire in Mme d'Aulnoy's Fairy Tale: La Chatte Blanche », Esprit Créateur, vol. XXIV, nº 3, 1989, p. 52-63.

HANNON, Patricia, Fabulous Identities. Women's Fairy Tales in Seventeenth-Century France, Amsterdam, Rodopi, 1998.

HOFFMANN, Kathryn A., « Matriarchal Desires and Labyrinths of the Marvelous: Fairy Tales by Old Regime Women », dans Colette H. Winn et Donna Kuizenga (éds.), Women Writers in Pre-Revolutionary France: Strategies of Emancipation, New York, Garland, 1997, p. 281-97.

LA FORCE, Rose-Charlotte de Caumont de, L'Enchanteur, 1697, dans Contes. Bibliothèque des Génies et des Fées, éd. cit., p. 339-355. [Raymonde Robert (éd.)]

LA FORCE, Rose-Charlotte de Caumont de, Persinette, 1697, dans Contes. Bibliothèque des Génies et des Fées, éd. cit., p. 331-338. [Raymonde Robert (éd.)]

LA FORCE, Rose-Charlotte de Caumont de., «Mademoiselle de La Force peinte par ellemême », Les Jeux de l'esprit ou La Promenade de Conti, 1701, Paris, Aubry, 1862.

LEGAULT, Marianne, Narrations déviantes: L'intimité entre femmes dans l'imaginaire français du dix-septième siècle, Laval, Presses de l'Université Laval, 2008.

L'HÉRITIER, Marie-Jeanne de Villandon, L'Adroite princesse ou les aventures de Finettte, 1696, dans Contes. Bibliothèque des Génies et des Fées, éd. cit., p. 93-114. [Raymonde Robert (éd.)] 
MAISTRE WELCH, Marcelle, «La Femme, le mariage et l'amour dans les contes de fées mondains du XVII ${ }^{\mathrm{e}}$ siècle français », Papers on French Seventeenth-Centure Literature, vol. $\mathrm{XX}, \mathrm{n}^{\circ} 18,1983$, p. 47-58.

MURAT, Henriette-Julie de, Anguillette, 1697, Madame de Murat. Contes. Bibliothèques des génies et des fées, Paris, Champion, 2006, t. 3, p. 85-117. [Geneviève Patard (éd.)]

MURAT, Henriette-Julie de, Peine Perdue, 1708, Contes de fées du Grand Siècle, New York, Columbia Press, 1934. [M. E. Storer (éd.)]

PERRAULT, Charles, Contes, Paris, Gallimard (Folio), 1981. [J.-P. Collinet (éd.)]

SEIFERT, Lewis C., Fairy Tales, Sexuality, and Gender in France, Cambridge, Cambridge University Press, 1996.

STORER, Mary Elizabeth, Un épisode littéraire de la fin du XVII siècle. La mode des contes de fées (1685-1700), Paris, Champion, 1928.

TIMMERMAN, Linda, L'accès des femmes à la culture sous l'Ancien Régime, Paris, Champion Classiques, 2005.

TRINQUET, Charlotte, « Happily Ever After? Not so Easily! Seventeenth-Century Fairy Tales and their Unconventional Endings », Papers on French Seventeenth-Century Literature, vol. XXXVII, no 72,2010 , p. 45-54.

VELLENGA, Carolyn, « Rapunzel's Desire: A Reading of Mlle de La Force », Merveilleux et contes, vol. VI, nº1, 1992, p. 59-73.

VERDIER, Gabrielle, « Approaches to the Literary Fairy Tale », dans Marie-France Hilgar (éd.), Actes de Las Vegas : théorie dramatique, Théophile de Viau, les contes de fées : actes du XXIIe colloque de la North American Society for Seventeenth-Century French Literature, University of Nevada, Las Vegas, 1-2 mars 1990, Tubingen, PFSCL, 1991, p. 141-146.

ZIPES, Jack, Fairy Tales and the Art of Subversion: The Classical Genre for Children and the Process of Civilization, London, Heinemann, 1983. 